Supporting Information

\title{
Nanoscale Polyion Complex Vesicles for Delivery of Cargo Proteins and Cas9 Ribonucleoprotein
}

\section{Complexes to Plant Cells}

Masaki Odahara ${ }^{1, *^{*}}$, Kenta Watanabe ${ }^{1, \dagger}$, Riku Kawasaki ${ }^{1,5}$, Kousuke Tsuchiya ${ }^{2 *}$, Ayaka Tateishi ${ }^{1}$, Yoko Motoda ${ }^{1,3}$, Takanori Kigawa ${ }^{3}$, Yutaka Kodama ${ }^{1,4}$ \& Keiji Numata ${ }^{1,2 *}$.

${ }^{1}$ Biomacromolecule Research Team, RIKEN Center for Sustainable Resource Science, Saitama, Japan.

${ }^{2}$ Department of Material Chemistry, Kyoto University, Kyoto, Japan.

${ }^{3}$ Laboratory for Cellular Structural Biology, RIKEN Center for Biosystems Dynamics Research, Yokohama, Japan.

${ }^{4}$ Center for Bioscience Research \& Education, Utsunomiya University, Utsunomiya, Japan.

These authors contributed equally to this work.

*e-mail: numata.keiji.3n@ kyoto-u.ac.jp (K.N.); masaki.odahara@riken.jp (M.O.);

tsuchiya.kosuke.3n@kyoto-u.ac.jp (K.T.) 


\section{Experimental section}

\section{Synthesis of two-armed and carboxylated oligolysines.}

Reagents. H-Lys(Boc)-OMe hydrochloride, 1-[3-(dimethylamino)propyl]-3ethylcarbodiimide hydrochloride (WSCI $\mathrm{HCl}$ ), and Boc-sarcosine-OH were purchased from Watanabe Chemical Industries (Hiroshima, Japan). $\mathrm{CuSO}_{4}$, sodium ascorbate, tris(3hydroxypropyltriazolymethyl)amine (THPTA), propargyl bromide, $p$-toluenesulphonyl chloride, potassium phthalimide, ethylenediaminetetraacetic acid dianhydride (EDTADA), 1hydroxybenzotriazole monohydrate ( $\mathrm{HOBt})$, and potassium tertiary butoxide were purchased from Tokyo Chemical Industry (Tokyo, Japan). Tetra(ethylene glycol) was purchased from Sigma Aldrich (Missouri, USA). Potassium hydroxide, chloroform, methanol, tetrahydrofuran, sodium hydroxide, trifluoroacetic acid (TFA), and papain were purchased from Fujifilm-Wako Chemical industry (Tokyo, Japan). Acetonitrile was purchased from Kanto Chemical Industry (Tokyo, Japan). KAibA peptides were synthesized by BSI-RIKEN.

Synthesis of oligolysine via chemoenzymatic synthesis. Synthesis of oligolysine has been reported elsewhere. ${ }^{1}$ Briefly, $1 \mathrm{M} \mathrm{H}$-Lys(Boc)-OMe was dissolved in $1 \mathrm{M}$ phosphate buffer $(\mathrm{PB} ; \mathrm{pH} 8.0)$ and maintained at $40^{\circ} \mathrm{C}$ with stirring. A papain solution $\left(10 \mathrm{mg} \mathrm{mL}^{-1}\right)$ was added to the warmed solution and stirred for $4 \mathrm{~h}$. Precipitates were washed with Milli-Q water and isolated via centrifugation $(9,000 \mathrm{xg})$ at $4^{\circ} \mathrm{C}$. Oligolysine(Boc)-OMe was analysed by matrix-assisted laser desorption Ionization time-of-flight mass spectrometry MALDI-TOF-MS). The resulting peptide was dissolved in dichloromethane (DCM) and TFA (1/1 in volume) and the mixture was stirred at $25^{\circ} \mathrm{C}$ for $24 \mathrm{~h}$. The deprotected oligolysine was obtained after precipitation in diethyl ether.

Synthesis of alkyne-modified tetraethylene glycol (alkyne-TEG). Tetra(ethylene glycol) (TEG) (36 mmol) and potassium tertiary butoxide $(49.6 \mathrm{mmol})$ were dissolved in dry tetrahydrofuran (THF, $70 \mathrm{~mL}$ ) and maintained at $0^{\circ} \mathrm{C}$ on ice for $30 \mathrm{~min}$. Propargyl bromide (36.5 mmol) in THF (10 mL) was added dropwise to the resulting solution. After $24 \mathrm{~h}$, solvent was evaporated and the pellet redissolved in chloroform. After extraction $(1 \mathrm{~N} \mathrm{HCl}$, twice; water, twice; brine, once) and drying with $\mathrm{MgSO}_{4}$, the product was purified by column chromatography (methanol:chloroform, 1:19). Synthesis was confirmed by ${ }^{1} \mathrm{H} \mathrm{NMR}$ in $\mathrm{CDCl}_{3}$. 
Synthesis of alkyne-TEG-Tos. Alkyne-TEG (21 mmol), triethylamine (63 mmol), and $p$ toluenesulfonyl chloride $(21 \mathrm{mmol})$ were dissolved in dry chloroform $(30 \mathrm{~mL})$ and stirred for 24 h. Extraction was carried out with water and brine. After drying with $\mathrm{MgSO}_{4}$, the compound was directly used for next reaction without any purification. Synthesis was confirmed by ${ }^{1} \mathrm{H}$ NMR in $\mathrm{CDCl}_{3}$.

Synthesis of alkyne-TEG-phthalimide. Alkyne-TEG-Tos $(12 \mathrm{mmol})$ was dissolved in dry $N, N$-dimethylformamide (DMF, $20 \mathrm{~mL}$ ) and maintained at $110^{\circ} \mathrm{C}$ in an oil bath. After $30 \mathrm{~min}$, potassium phthalimide $(17.8 \mathrm{mmol})$ was added to the resulting solution and stirred for $24 \mathrm{~h}$. Filtration was carried out to remove salts and the solvent was removed by aspiration. The resulting product was re-dissolved in chloroform and extraction was carried out using $1 \mathrm{~N} \mathrm{HCl}$, water, and brine. After drying with $\mathrm{MgSO}_{4}$, the product was purified by column chromatography (methanol:chloroform, 1:19). Synthesis was confirmed by ${ }^{1} \mathrm{H}$ NMR in $\mathrm{CDCl}_{3}$.

Synthesis of alkyne-TEG-NH2. Alkyne-TEG-phthalimide $(9.6 \mathrm{mmol})$ and hydrazine hydrochloride $(96 \mathrm{mmol})$ were dissolved in methanol $(10 \mathrm{~mL})$ and stirred for $24 \mathrm{~h}$. After removal of salts by filtration using a celite, solvent was evaporated and the crude product was redissolved in chloroform. The organic layer was washed with water and brine. After drying with $\mathrm{MgSO}_{4}$, the product was purified by column chromatography (methanol:chloroform, 1:19). Synthesis was confirmed by ${ }^{1} \mathrm{H}$ NMR in $\mathrm{CDCl}_{3}$.

Synthesis of Boc-protected two-armed oligolysine. Oligolysine(Boc)-OMe $(14 \mu \mathrm{mol})$ was dissolved in dried DMF $(2 \mathrm{~mL})$ at $0^{\circ} \mathrm{C}$ on ice for $30 \mathrm{~min}$. EDTADA $(7 \mu \mathrm{mol})$ was added to the resulting solution and stirred at for $24 \mathrm{~h}$. The product was precipitated against diethyl ether and redissolved in methanol. Dialysis of the peptide was carried out to purify the compound (MWCO 2,000). Afterward, the peptide $(10 \mu \mathrm{mol})$ and alkyne-TEG-NH $2(20 \mu \mathrm{mol})$ was dissolved in DMF $(2 \mathrm{~mL})$ and triethylamine $(22 \mu \mathrm{mol}), \mathrm{HOBt}(22 \mu \mathrm{mol})$, and WSCI HCl $(22 \mu \mathrm{mol})$ were added. The resulting mixture was stirred at $25^{\circ} \mathrm{C}$ for $24 \mathrm{~h}$. After isolation via precipitation in diethyl ether, Boc-protected two-armed oligolysine was obtained. Synthesis was confirmed by ${ }^{1} \mathrm{H}$ NMR in DMSO- $d_{6}$.

Synthesis of two-armed oligolysine. Boc-protected two-armed oligolysine $(10 \mu \mathrm{mol})$ was dissolved in DMF and TFA ( $2 \mathrm{~mL}, 1 / 1$ in volume) and the mixture was stirred at $25^{\circ} \mathrm{C}$. After 24 $\mathrm{h}$, the product was isolated via precipitation against diethyl ether. After evaporation of the solvent, synthesis was confirmed by ${ }^{1} \mathrm{H}$ NMR in DMSO- $d_{6}$. 
Synthesis of carboxylated oligolysine. Oligolysine(Boc)-OMe (14 $\mu \mathrm{mol})$ was dissolved in dried DCM and TFA ( $2 \mathrm{~mL}, 1 / 1$ in volume). The mixture was stirred at $25^{\circ} \mathrm{C}$ for $24 \mathrm{~h}$. The product was isolated via precipitation against diethyl ether to yield oligolysine-OMe after drying. Oligolysine-OMe $(10 \mu \mathrm{mol})$ and succinic anhydride $(100 \mu \mathrm{mol})$ were then dissolved in dry DMF $(2 \mathrm{~mL})$ and stirred for $24 \mathrm{~h}$. The product was precipitated against diethyl ether and isolated via centrifugation $(9,000 \mathrm{x} g)$ at $4^{\circ} \mathrm{C}$. Synthesis was confirmed by ${ }^{1} \mathrm{H}$ NMR in DMSO- $d_{6}$.

Plant materials and growth conditions. Arabidopsis thaliana (accession Col-0) was used. The growth media used for A. thaliana were as follows: germination medium (GM), half-strength Murashige and Skoog (MS) Basal Medium powder (Sigma Aldrich, Missouri, USA) supplemented with $5 \mathrm{~g} \mathrm{~L}^{-1}$ sucrose, callus induction medium (CIM), and Gamborg's B-5 Basal Medium with Minimal Organics fine powder (Sigma Aldrich) supplemented with $20 \mathrm{~g} \mathrm{~L}^{-1}$ glucose, $0.5 \mathrm{mg} \mathrm{L}^{-1}$ 2,4-dichlorophenoxyacetic acid (Fujifilm-Wako Chemical industry, Tokyo, Japan), and $50 \mu \mathrm{g} \mathrm{L}^{-1}$ kinetin (Sigma Aldrich). All growth media were adjusted to $\mathrm{pH} 5.7$ with aqueous $\mathrm{KOH}$ and solidified with $0.3 \mathrm{~g} \mathrm{~L}^{-1}$ phytagel.

Calli were prepared as previously described with slight modifications. ${ }^{2} A$. thaliana seeds were surface sterilized with $70 \%$ ethanol and $2 \%$ sodium hypochlorite and then rinsed five times with autoclaved Milli-Q purified water. The surface-sterilized seeds were soaked in Milli-Q purified water at $4^{\circ} \mathrm{C}$ in the dark for $3 \mathrm{~d}$ and then sown on GM medium and grown in the dark (21 $\pm 1^{\circ} \mathrm{C}$ ). Hypocotyls from 7 -d-old seedlings were harvested and plated onto CIM medium in the dark $\left(21 \pm 1{ }^{\circ} \mathrm{C}\right)$. After incubation on CIM medium for $8 \mathrm{~d}$, calli formed from the hypocotyls and were used for further studies.

Formulation of PICsomes. The oligopeptide derivatives two-armed oligolysine and carboxylated oligolysine were synthesized as shown in the synthesis section above. Solutions of two-armed oligolysine and carboxylated oligolysine were mixed at 250 and $500 \mu \mathrm{M}$, respectively, and vortexed for $10 \mathrm{s.}^{3}$ Their size distribution was measured by dynamic light scattering measurements (DLS; Zetasizer Nano ZS, Malvern Instruments Co. Ltd., Worcestershire, UK) 30 
min after vortexing. Morphological observations were performed by field emission scanning electron microscopy (FE-SEM; Carl Zeiss, Oberkochen, Germany). Samples (1 $\mu \mathrm{L})$ were placed on silicon wafers, and the solvent was allowed to completely dry under vacuum for $24 \mathrm{~h}$. The samples were observed without staining, with acceleration voltage and working distance fixed at $2.0 \mathrm{kV}$ and $2.0 \mathrm{~mm}$, respectively.

Preparation of cross-linked PICsomes. After formulating the PICsome, we added 1-(3dimethylaminopropyl)-3-ethylcarbodiimide (EDC, $5 \mathrm{mM}$ ) to the resulting suspension and incubated the mixtures for $3 \mathrm{~h}$ to allow cross-linking to proceed. The size distribution and morphological observations were then verified by DLS and FE-SEM, respectively. After purification by dialysis for $24 \mathrm{~h}$, the cross-linked ratio was calculated by ${ }^{1} \mathrm{H}$ nuclear magnetic resonance (NMR). The hollow structure of PICsomes was confirmed by preparing ultrathin sections embedded in EPON resin as follows. The samples were placed and embedded in lowmelting agarose gels. The hydrogels were gradually dried in a graded methanol series $(25 \%, 50 \%$, $75 \%, 90 \%, 99 \%, 100 \%$ ), followed by propylene oxide, at which point the solvent was completely replaced with EPON resin. Afterwards, the samples were placed on sample grids and the resin induced to solidify by heating at $60^{\circ} \mathrm{C}$ for $24 \mathrm{~h}$. Ultrathin sections were prepared with an ultramicrotome and completely dried on a heater at $40^{\circ} \mathrm{C}$. Finally, the samples were coated with carbon for $30 \mathrm{~min}$ and observed by FE-SEM.

CPP modification via click chemistry. After preparation of cross-linked PICsomes, we added $\mathrm{KAibA}_{3} \mathrm{~N}_{3}, \mathrm{CuSO}_{4}$, and tris-hydroxypropyltriazolylmethylamine (THPTA) for the azidealkyne click reaction at concentrations of 100,20, and $120 \mu \mathrm{M}$, respectively, and sodium ascorbate at $500 \mu \mathrm{M}$ to the cross-linked PICsome suspension. After the dialysis with Milli-Q to remove the non-reacted peptides and chemicals, we determined the size distribution and performed morphological observations by DLS and FE-SEM, respectively. Modification of CPP to PICsomes was evaluated by reverse phase-HPLC. Boc-Gly-OH was added as an internal standard. 
The mobile phase were mixture of water and acetonitrile and the absorbance at $260 \mathrm{~nm}$ was monitored to detect molecules.

Evans blue assay. PICsome derivatives (500 $\mu \mathrm{M}$ in water) were infiltrated into calli via the vacuum and compression method (-0.08 MPa, $1 \mathrm{~min} ;+0.08 \mathrm{MPa}, 1 \mathrm{~min}) .{ }^{4}$ After 24 and $48 \mathrm{~h}$ incubation, we stained calli with Evans blue staining solution according to a previously described method with slight modifications..$^{5}$ The calli were washed with Milli-Q water and then incubated with $50 \mu \mathrm{g} \mathrm{mL}^{-1}$ Evans blue for $10 \mathrm{~min}$ to stain dead cells. The calli were then rinsed three times with Milli-Q water and soaked in 50\% methanol containing 1\% SDS solution for $2 \mathrm{~h}$. After centrifugation of the lysates, the absorbance of the supernatants at $600 \mathrm{~nm}$ was measured. As a positive control, we stained calli that had been soaked in $70 \%$ ethanol for 60 min to kill all cells before Evans blue staining. Four biological replicates per condition were used. The absorbance was normalized to the callus protein concentration, as determined by Bradford assay.

Complexation of Citrine. Citrine was synthesized via in vitro cell-free synthesis. ${ }^{6,7}$ Twoarmed peptides and Citrine $(1 \mu \mathrm{g})$ were mixed first and then carboxylated oligolysine was added and the resulting solution was mixed by vortexing for $10 \mathrm{~s}$. After $10 \mathrm{~min}$, EDC solution was added at concentration of $5 \mathrm{mM}$, and the mixture was incubated for $3 \mathrm{~h}$. KAibA- $\mathrm{N}_{3}$ peptides were then added to PICsomes via the azide-alkyne click reaction. The complexation was performed with dialysis to remove free Citrine. The size distribution was measured by DLS. Complexation was confirmed by fluorescence correlation spectroscopy (FCS; Carl Zeiss LSM880). ${ }^{8}$ The Citrineloaded PICsomes were excited with $488 \mathrm{~nm}$ in order to obtain a maximal signal to noise ratio. One hundred microliters of the Citrine-loaded PICsome solution was applied to a glass bottom plate above the immersion objective. Fluorescence emission was collected through the same objective and finally detected by highly sensitive avalanche photodiodes. An autocorrelation function $(G(t))$ was obtained from the fluorescence intensity fluctuations. Complexed Citrine was quantified by silver staining. After incubation with sample buffer solution containing 5\% mercaptoethanol at 
$70^{\circ} \mathrm{C}$ for 7 min, denatured Citrine standard solution or Citrine@PICsome was loaded in 10\% acrylamide gel. Then, SDS-PAGE was carried out and the resulting gel was stained with the silver stain plus kit (Bio-Rad Laboratories, California, USA). Ratio for Citrine encapsulated in the PICsomes was calculated by dividing concentration of Citrine after complexation and subsequent dialysis by that before complexation and dialysis.

Preparation and complexation of RNP complex. The EnGen sgRNA Synthesis Kit, $S$. pyogenes (New England BioLabs, Massachusetts, USA), was used to synthesize gRNAs, which were then purified with Clean \& Concentrator ${ }^{\mathrm{TM}}-25$ (Zymo Research, California, USA) according to the manufacturer's protocol. Primers for sgRNA synthesis are shown in Table S5. Alt-R ${ }^{\circledR}$ Cas 9 Nuclease V3 (Cas9; Integrated DNA Technology, Iowa, USA; $200 \mu \mathrm{M}$ ), which includes nuclear localization sequences (NLSs), and $200 \mu \mathrm{M}$ of each purified gRNA were mixed and incubated at $25^{\circ} \mathrm{C}$ for $10 \mathrm{~min}$ to form an RNP complex. The formation of the complexes with CPP-displayed cross-linked PICsome and their confirmation were carried out by the same method as for Citrine. For confocal laser-scanning microscopy (CLSM, Carl Zeiss LSM880) observations, the Cas9 protein was fluorescently labelled with rhodamine B isothiocyanate (Sigma-Aldrich) according to a previously described method ${ }^{6}$ prior to the formation of the RNP complex. Briefly, $10 \mathrm{mg} \mathrm{mL}^{-1}$ of rhodamine B isothiocyanate solution in dimethyl sulfoxide was dropwise added to the Cas9 solution under gentle stirring, followed by incubation at $4^{\circ} \mathrm{C}$ overnight with continuous stirring. The free rhodamine B isothiocyanate was removed by dialysis. For editing of the nuclear genome with RNPs, gRNA1 and gRNA2 were mixed with Cas9 and loaded into CPP-displayed crosslinked PICsomes.

In vitro DNA cleavage test with RNP complex. To check the efficiency of the gRNAs, an in vitro DNA cleavage test was performed. A 459-bp PCR fragment from PDS3 was amplified using A. thaliana genomic DNA template and specific primers (Table S5). The PCR product and the RNP complex at concentrations of 46 and $300 \mathrm{nM}$, respectively, were mixed in $1 \times$ NEBuffer 
3.1 (New England BioLabs), incubated for $15 \mathrm{~min}$ at $37^{\circ} \mathrm{C}$, and then analysed by agarose gel electrophoresis.

Introduction of Citrine and RNP complex into A. thaliana calli. To introduce Citrine or PICsome into cells, A. thaliana calli ( $8 \mathrm{~d}$ after callus induction) were soaked in various buffers containing Citrine alone, Citrine complexed with cross-linked PICsome, or Citrine complexed with CPP-displayed cross-linked PICsome and then subjected to the vacuum and compression method (-0.08 MPa, $1 \mathrm{~min} ;+0.08 \mathrm{MPa}, 1 \mathrm{~min}$ ) and subsequent incubation for $30 \mathrm{~min}^{4}$. A similar series of experiments was also performed for the introduction of the rhodamine B-labelled RNP and RNP complexes. Treated calli were then plated on GM medium for $24 \mathrm{~h}$. Internalization of Citrine and rhodamine B-labelled RNP complexes was confirmed by CLSM (LSM880, Carl Zeiss).

Detection of nuclear mutations. Genomic DNA was extracted from treated calli by a brief extraction method. ${ }^{9}$ To prepare sequencing libraries, we followed a two-step PCR approach. In the first PCR, a 212-bp DNA segment encompassing the target sites of the gRNAs was amplified for 25 cycles, using the indicated primers with a universal adapter sequence (Table S5) and KAPA HiFi HotStart ReadyMix (Kapa Biosystems, Massachusetts, USA), with the extracted genomic DNA as templates. The amplicons from the first PCR were then subjected to a second PCR for eight cycles using the Nextera XT Index Kit v2 Set (Illumina, California, USA) to ligate adapters. The libraries from each sample were indexed and mixed, and then sequenced on an Illumina MiSeq platform with MiSeq Reagent Kit v2 (300 cycles, Illumina). Each read was aligned to the PDS target sequence using strand NGS v3.4 software (Strand Life Sciences, Bangalore, India). Aligned reads with a quality score below $30(Q<30)$ were discarded using the strand NGS software. We then trimmed the reads to 20 nucleotides on either side of the predicted double-stranded break site targeted by gRNAs using the strand NGS software. Only Indels in the vicinity of the target sites were scored as a mutation. The mutation rate was calculated based on the number of mutant reads/total read count. 
Chemoenzymatic synthesis of Boc-protected oligolysine

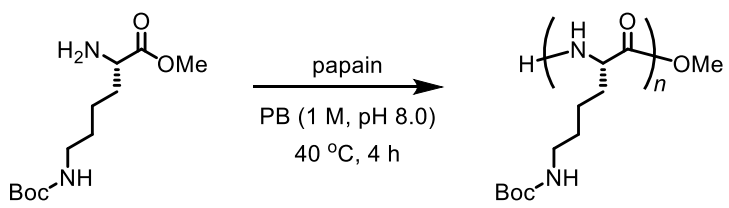

Synthesis of TEG segment

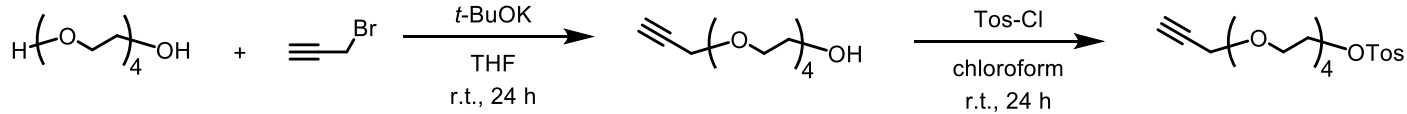

$$
\begin{aligned}
& \underset{110^{\circ} \mathrm{C}, 24 \mathrm{~h}}{\mathrm{DMF}}
\end{aligned}
$$

Synthesis of cationic two-armed oligolysine<smiles>CC(C)N[C@@H](CCCCNC(=O)OCc1ccccc1)C(=O)C(C)(C)C(C)(C)C</smiles><smiles>CCC(C)(C)C(=O)C(CCCCNC(=O)OC(C)(C)C)NC(C)(C)C(=O)CN(CCN(CC(=O)O)CC(=O)N[C@@H](CCCCNC(=O)OC(C)(C)C)C(=O)OC)CC(=O)O</smiles>

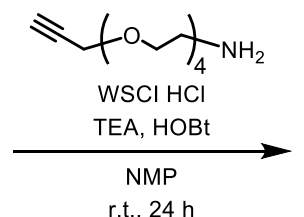

r.t., $24 \mathrm{~h}$

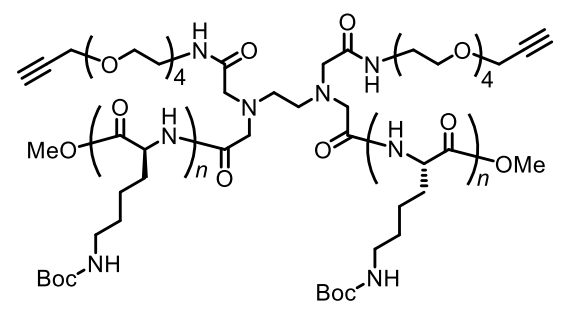

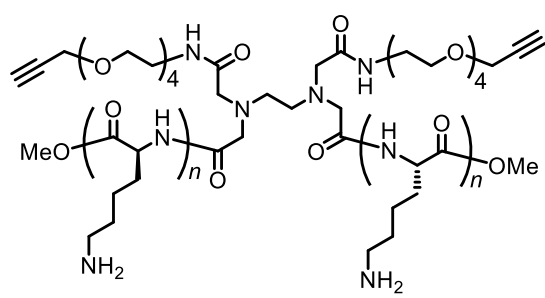

two-armed oligolysine

Synthesis of anionic carboxylated oligolysine<smiles>[M]OC(C)(C)C(=O)C(CCCCNC(=O)OCc1ccccc1)NC(C)C</smiles><smiles>CCCC(C)C</smiles><smiles>COC(C)(C)C(=O)[C@H](CCCCN)NC(C)C</smiles><smiles>CC(C)CCCC(=O)OC(=O)CCC(C)(F)F</smiles><smiles>COC(C)(C)C(=O)[C@H](CCCCNC(=O)CCC(=O)O)NC(C)C</smiles>

carboxylated oligolysine 
Figure S1. Synthesis of two-armed and carboxylated oligolysine.

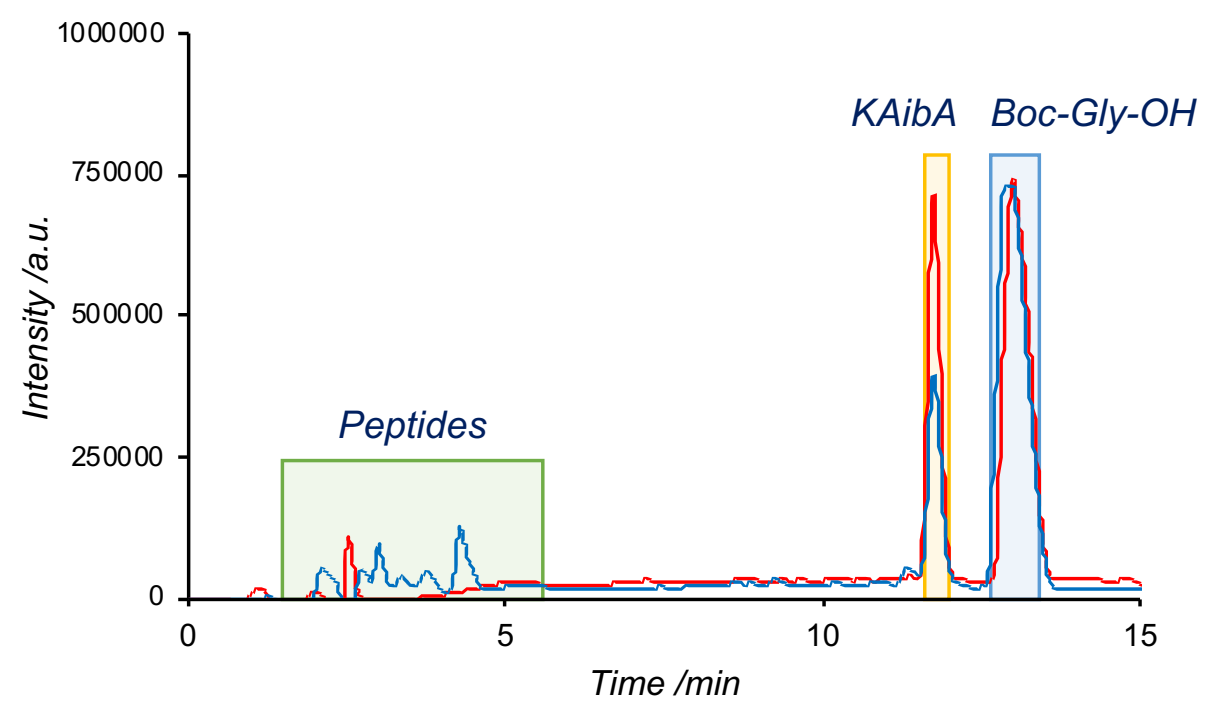

Figure S2. Representative elusion curve of peptide solution. KAibA peptides (Red curve) or KAibA peptides modified PICsome (Blue curve) were analyzed by reverse phase HPLC. BocGly-OH was added as an internal standard. The mobile phase were mixture of water and acetonitrile and the absorbance at $260 \mathrm{~nm}$ was monitored to detect molecules. 


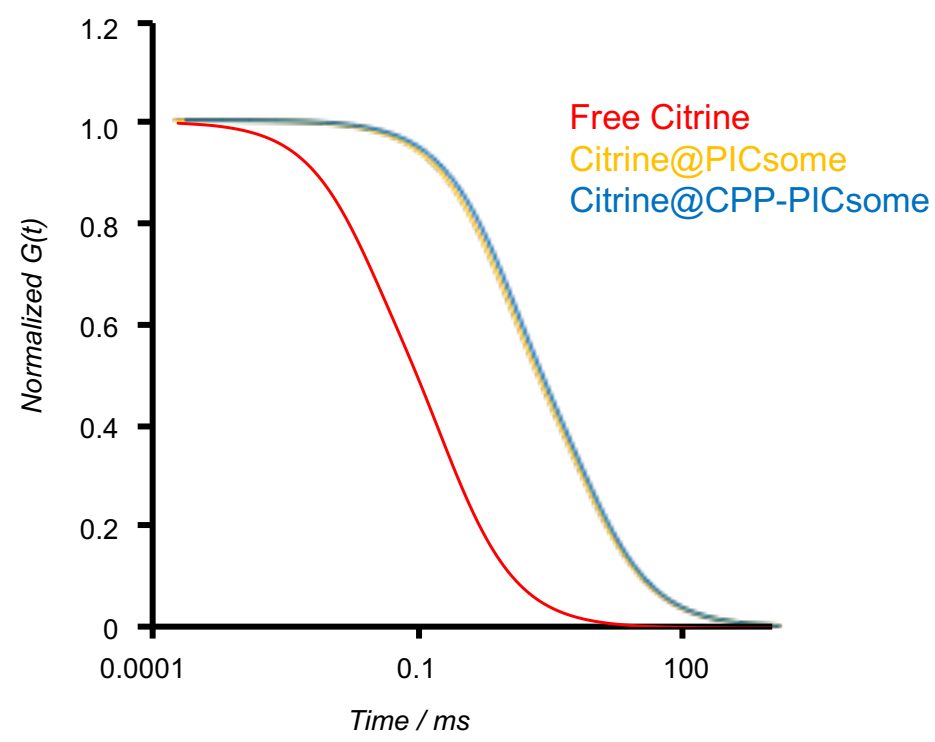

Figure S3. Fluorescence correlation spectra from Citrine. Citrine was encapsulated in PICsome (Citrine, $1 \mu \mathrm{g}$; PICsome, $500 \mu \mathrm{M}$ ) that was then cross-linked (Citrine@PICsome) and modified with CPP (Citrine@CPP-PICsome). After removal of excess EDC and CPP, FCS measurements were carried out. $G(t)$ indicates the autocorrelation function, which reflects the time-scales of the fluorescence intensity fluctuations. 

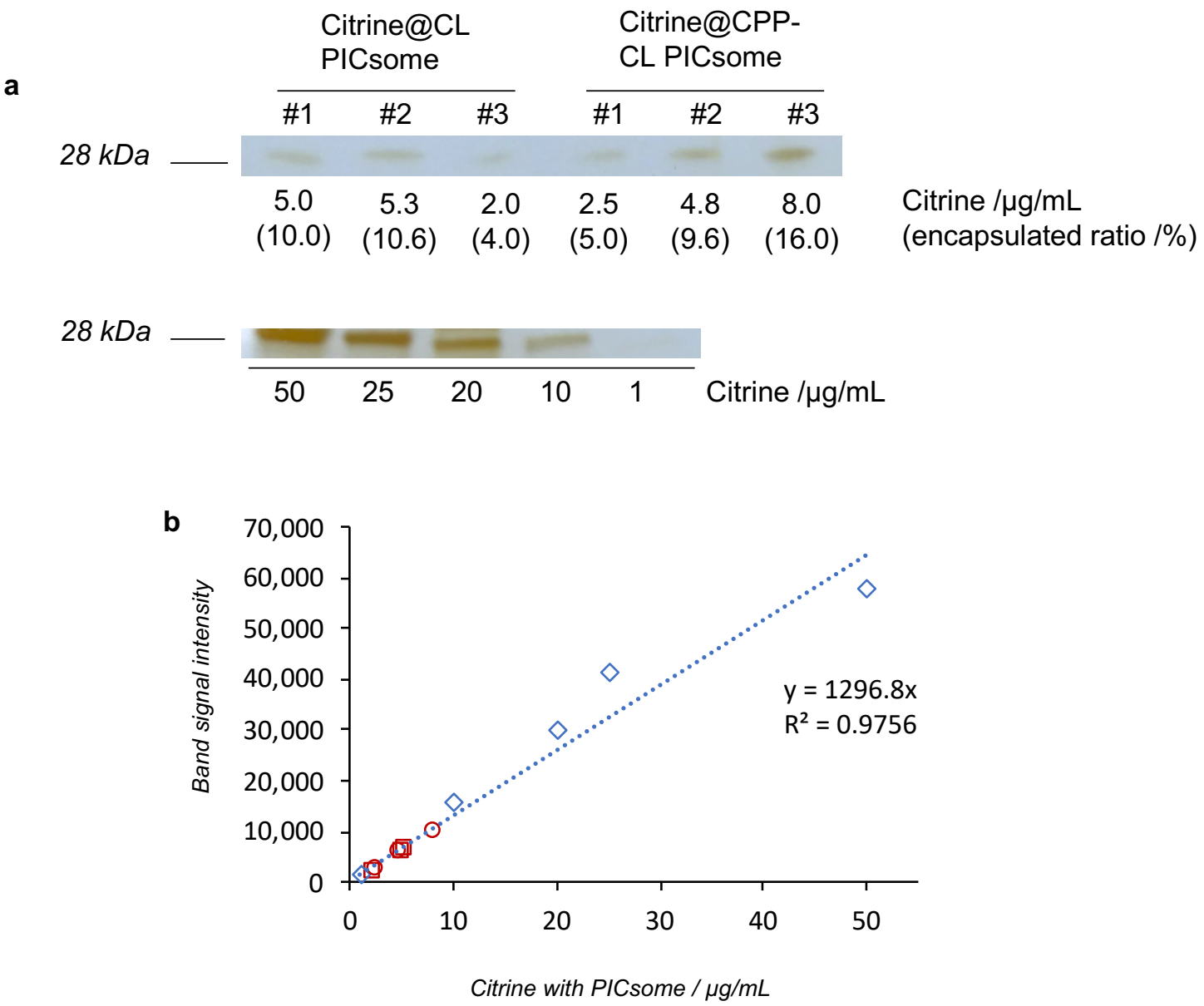

Figure S4. Quantification of complexed Citrine. (a) Silver staining images of SDS-PAGE gels for Citrine quantification. After SDS-PAGE, the gels were stained with silver staining solution. Complexed Citrine (upper panel) was quantified against a standard curve (b) from Citrine standards (lower panel). Quantified concentrations and encapsulated efficiency are shown under the bands. (b) Standard curve for Citrine quantification. Blue diamonds, Citrine standards; red squares, Citrine@CL-PICsome $(N=3)$; red circles, Citrine@CPP-PICsome $\left(N_{-}=3\right)$. The band signals were measured in ImageJ. 

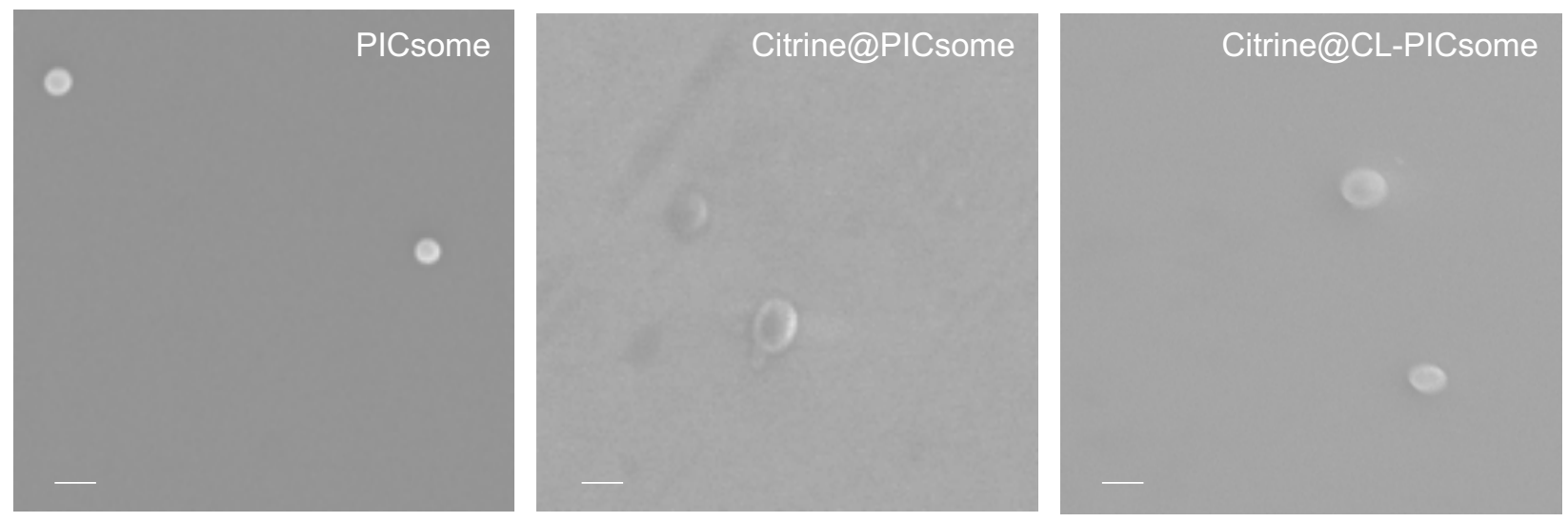

Figure S5. Representative morphology of Citrine@PICsome derivatives. PICsomes encapsulating Citrine were observed by FE-SEM (working distance, $1.4 \mathrm{~mm}$; acceleration voltage, $2.0 \mathrm{kV}$ ). Scale bars, $200 \mathrm{~nm}$. An image of Citrine@CPP-PICsome is shown in Figure 2e. 


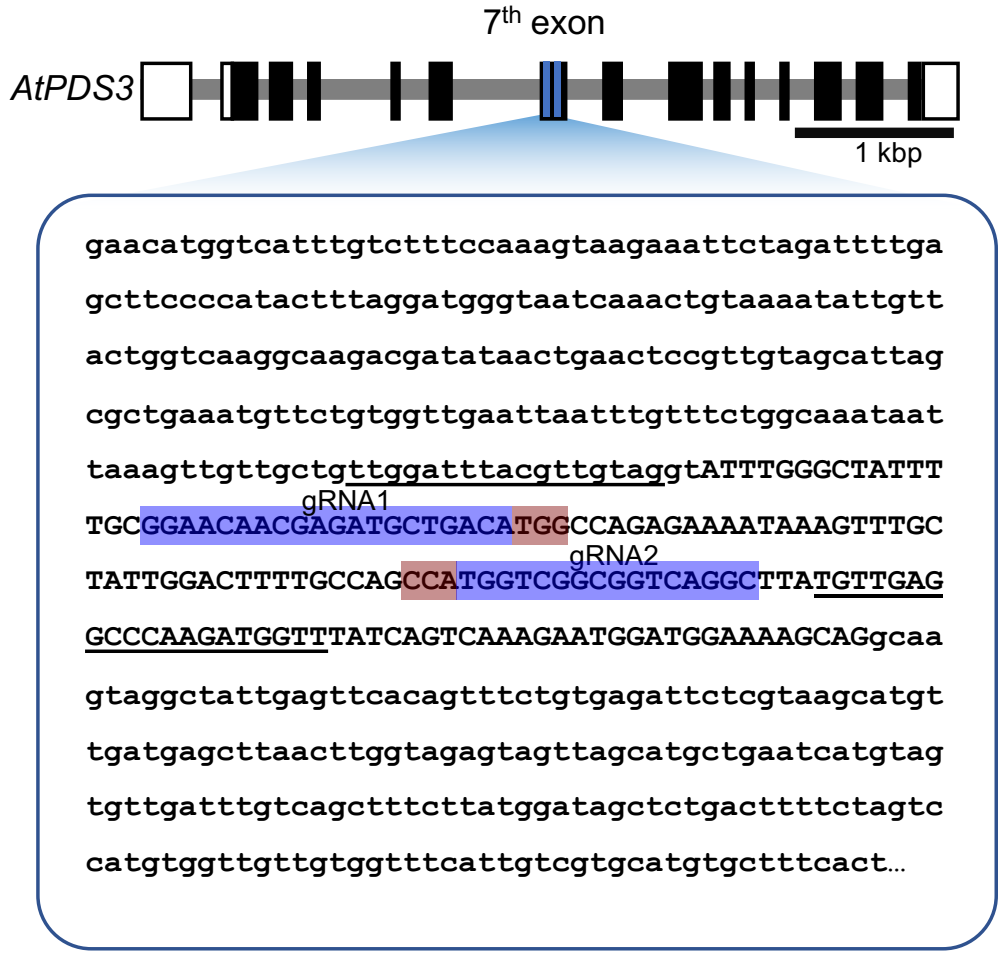

b

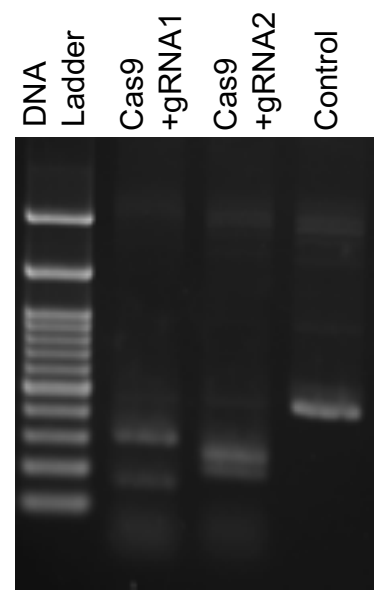

Figure S6. Guide RNAs used in this study. (a) Schematic representation of A. thaliana PDS3 target sequences. White and black boxes indicate untranslated region and exons, respectively. Gray bars indicate introns. The sequence of the $7^{\text {th }}$ exon and neighbouring introns are shown in uppercase and lowercase letters, respectively. The 20-bp target sequences are highlighted in blue, and the PAM sequences (NGG or CCN) are highlighted in red. Underlined letters indicate primer sequences for deep sequencing analysis. (b) In vitro cleavage assay for the two gRNAs. PDS3 fragments (459 bp) amplified using A. thaliana genomic DNA as template and specific primers (Table S5) were incubated with RNP complexes. Predicted sizes for the cleaved fragments are 160 and $299 \mathrm{bp}$ for gRNA1, and 205 and $254 \mathrm{bp}$ for gRNA2. DNA ladder corresponds to $3 \mathrm{kbp}, 1 \mathrm{kbp}$, 900 bp, 800 bp, 700 bp, 600 bp, 500 bp, 400 bp, 300 bp, 200 bp, and 100 bp.

Table S1. Solution properties of PICsome, cross-linked PICsome (CL-PICsome), and CPPmodified PICsome (CPP-PICsome). 


\begin{tabular}{lcll}
\hline & $D_{H}(\mathrm{~nm})^{1}$ & PDI $^{2}$ & $\zeta$-potential $(\mathrm{mV})$ \\
\hline PICsome & $169 \pm 9$ & $0.15 \pm 0.08$ & $-1.2 \pm 0.4$ \\
CL-PICsome & $155 \pm 3$ & $0.18 \pm 0.02$ & $-2.3 \pm 1.2$ \\
CPP-PICsome & $168 \pm 2$ & $0.13 \pm 0.01$ & $+24.3 \pm 2.5$ \\
& & & \\
\hline PICsomes were prepared in aqueous media (cationic peptide, $250 \mu \mathrm{M}$; anionic peptide, $500 \mu \mathrm{M})$.
\end{tabular}
Measurements were carried out in water ( $\mathrm{pH} 7.4)$ and temperature was fixed at $25^{\circ} \mathrm{C}$. Data are represented as mean \pm s.d. $(N=3)$. $\zeta$-Potential was measured using a capillary cell.

${ }^{1} D_{H}$ : diameter. ${ }^{2}$ PDI: polydispersity index. 
Table S2. Solution properties of Citrine@PICsome, Citrine@CL-PICsome, and Citrine@CPPPICsome.

\begin{tabular}{|c|c|c|c|c|}
\hline & $D_{H}(\mathrm{~nm})^{1}$ & $\mathrm{PDI}^{2}$ & $\begin{array}{c}\zeta \text {-potential } \\
(\mathrm{mV})\end{array}$ & $\begin{array}{l}\text { Diffusion time of } \\
\text { Citrine }(\mu \mathrm{s})\end{array}$ \\
\hline Citrine & NA & NA & NA & 115 \\
\hline \multicolumn{5}{|l|}{ Citrine@ } \\
\hline & $175 \pm 4$ & $0.11 \pm 0.04$ & $-0.4 \pm 0.2$ & 2830 \\
\hline \multicolumn{5}{|l|}{ PICsome } \\
\hline \multicolumn{5}{|l|}{ Citrine@ } \\
\hline & $152 \pm 3$ & $0.12 \pm 0.02$ & $-0.5 \pm 0.3$ & 2850 \\
\hline \multicolumn{5}{|l|}{ CL-PICsome } \\
\hline Citrine@CPP-PICsome & $160 \pm 4$ & $0.13 \pm 0.02$ & $+25.2 \pm 3.0$ & 2840 \\
\hline \multicolumn{5}{|c|}{ PICsomes were prepared in aqueous media (cationic peptide, $250 \mu \mathrm{M}$; anionic peptide, $500 \mu \mathrm{M}$ ). } \\
\hline \multicolumn{5}{|c|}{$\begin{array}{l}\text { Measurements were carried out in water ( } \mathrm{pH} 7.4) \text { and temperature was fixed at } 25^{\circ} \mathrm{C} \text {. Data are } \\
\text { represented as mean } \pm \text { s.d. }(N=3 \text { ). } \zeta \text {-Potential was measured using a capillary cell. Diffusion time } \\
\text { was determined by FCS measurement using confocal laser-scanning microscopy. }\end{array}$} \\
\hline
\end{tabular}


Table S3. Solution properties of RNP@PICsomes.

\begin{tabular}{|c|c|c|c|c|}
\hline & $\mathrm{RNP}(\mu \mathrm{M})$ & $D_{H}(\mathrm{~nm})^{1}$ & $\mathrm{PDI}^{2}$ & Diffusion time of RNP $(\mu \mathrm{s})$ \\
\hline RNP & 0.1 & NA & NA & 800 \\
\hline RNP@PICsome & 0.1 & $204 \pm 0.4$ & 0.08 & NA \\
\hline RNP@CL-PICsome & 0.1 & $210 \pm 4$ & 0.21 & 2850 \\
\hline RNP@CPP-PICsome & 0.1 & $217 \pm 5$ & 0.24 & 2890 \\
\hline RNP@PICsome & 0.31 & $208 \pm 8$ & 0.25 & NA \\
\hline RNP@CL-PICsome & 0.31 & $254 \pm 11$ & 0.22 & 2200 \\
\hline RNP@CPP-PICsome & 0.31 & $284 \pm 13$ & 0.28 & NA \\
\hline RNP@PICsome & 0.62 & $278 \pm 12$ & 0.27 & NA \\
\hline RNP@CL-PICsome & 0.62 & $295 \pm 9$ & 0.12 & 1820 \\
\hline RNP@CPP-PICsome & 0.62 & $314 \pm 11$ & 0.26 & NA \\
\hline
\end{tabular}

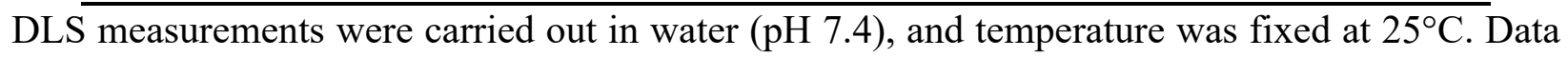
are represented as mean \pm s.d. $(N=3)$. $\zeta$-Potential was measured using a capillary cell. Diffusion time of rhodamine B-labelled RNP was measured by FCS. NA: not applicable. ${ }^{1} D_{H}$ : diameter. ${ }^{2}$ PDI: polydispersity index. 
Table S4. Deep sequencing data for mutation detection.

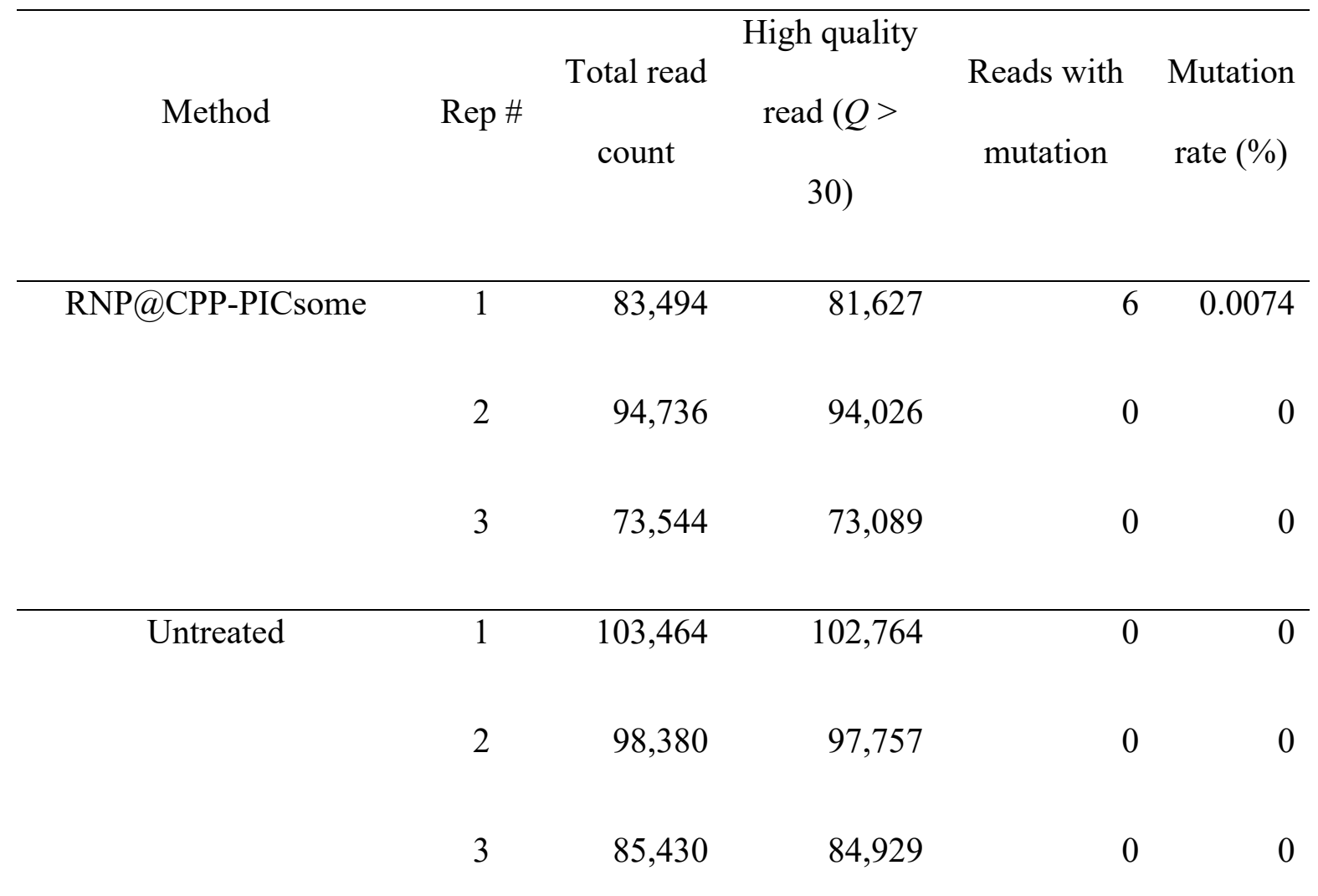

Number of reads with mutation in the vicinity of the double-stranded break site was counted and corrected for background. 
Table S5. Primers used in this study

\begin{tabular}{|c|c|c|}
\hline Name & Purpose & Sequence $\left(5^{\prime}-3^{\prime}\right)$ \\
\hline \multirow[t]{2}{*}{038} & gRNA & TTCTAATACGACTCACTATAGGAACAACGAGATGCTGACA \\
\hline & synthesis 1 & GTTTTAGAGCTAGA \\
\hline \multirow[t]{2}{*}{039} & gRNA & TTCTAATACGACTCACTATAGCCTGACCGCCGACCATGGCG \\
\hline & synthesis 2 & TTTTAGAGCTAGA \\
\hline \multirow[t]{3}{*}{001} & In vitro & TTACTGGTCAAGGCAAGACGATA \\
\hline & cleavage test & \\
\hline & Fw & \\
\hline \multirow[t]{3}{*}{002} & In vitro & AGTGAAAGCACATGCACGACA \\
\hline & cleavage test & \\
\hline & $\mathrm{Rv}$ & \\
\hline \multirow[t]{2}{*}{192} & NGS analysis & TCGTCGGCAGCGTCAGATGTGTATAAGAGACAGTGTTGCTG \\
\hline & Fw & TTGGATTTACGTTGT \\
\hline \multirow[t]{2}{*}{193} & NGS analysis & GTCTCGTGGGCTCGGAGATGTGTATAAGAGACAGAACCAT \\
\hline & Rv & CTTGGGCCTCAACA \\
\hline
\end{tabular}




\section{REFERENCES}

1. Miyamoto, T.; Tsuchiya, K.; Numata, K. Block Copolymer/Plasmid DNA Micelles Postmodified with Functional Peptides via Thiol-Maleimide Conjugation for Efficient Gene Delivery into Plants. Biomacromolecules 2019, 20, (2), 653-661.

2. Akama, K.; Shiraishi, H.; Ohta, S.; Nakamura, K.; Okada, K.; Shimura, Y. Efficient transformation of $<\mathrm{i}>$ Arabidopsis thaliana $</ \mathrm{i}\rangle$ : comparison of the efficiencies with various organs, plant ecotypes and $<\mathrm{i}>$ Agrobacterium $</ \mathrm{i}>$ strains. Plant Cell Rep. 1992, 12, (1), 7-11. 3. Koide, A.; Kishimura, A.; Osada, K.; Jang, W.-D.; Yamasaki, Y.; Kataoka, K. Semipermeable Polymer Vesicle (PICsome) Self-Assembled in Aqueous Medium from a Pair of Oppositely Charged Block Copolymers: Physiologically Stable Micro-/Nanocontainers of WaterSoluble Macromolecules. J. Am. Chem. Soc. 2006, 128, (18), 5988-5989.

4. Midorikawa, K.; Kodama, Y.; Numata, K. Vacuum/Compression Infiltration-mediated Permeation Pathway of a Peptide-pDNA Complex as a Non-Viral Carrier for Gene Delivery in Planta. Sci. Rep. 2019, 9, (1), 1-10.

5. $\quad$ Numata, K.; Horii, Y.; Oikawa, K.; Miyagi, Y.; Demura, T.; Ohtani, M. Library screening of cell-penetrating peptide for BY-2 cells, leaves of Arabidopsis, tobacco, tomato, poplar, and rice callus. Sci. Rep. 2018, 8, (1), 1-17.

6. $\quad \mathrm{Ng}, \mathrm{K}$. K.; Motoda, Y.; Watanabe, S.; Sofiman Othman, A.; Kigawa, T.; Kodama, Y.; Numata, K. Intracellular Delivery of Proteins via Fusion Peptides in Intact Plants. Plos One 2016, 11, (4), e0154081-e0154081.

7. Spirin, A.; Baranov, V.; Ryabova, L.; Ovodov, S.; Alakhov, Y. A continuous cell-free translation system capable of producing polypeptides in high yield. Science 1988, 242, (4882), 1162-1164.

8. Anraku, Y.; Kishimura, A.; Oba, M.; Yamasaki, Y.; Kataoka, K. Spontaneous Formation of Nanosized Unilamellar Polyion Complex Vesicles with Tunable Size and Properties. J. Am. Chem. Soc. 2010, 132, (5), 1631-1636.

9. Edwards, K.; Johnstone, C.; Thompson, C. A simple and rapid method for the preparation of plant genomic DNA for PCR analysis. Nucleic Acids Res. 1991, 19, (6), 1349-1349. 